\title{
A Counting Proof for When 2 Is a Quadratic Residue
}

\section{Karthik Chandrasekhar, Richard Ehrenborg \& Frits Beukers}

To cite this article: Karthik Chandrasekhar, Richard Ehrenborg \& Frits Beukers (2020) A Counting Proof for When 2 Is a Quadratic Residue, The American Mathematical Monthly, 127:8, 750-751, DOI: $10.1080 / 00029890.2020 .1790925$

To link to this article: https://doi.org/10.1080/00029890.2020.1790925

曲 Published online: 21 Sep 2020.

Submit your article to this journal $\pi$

Llll Article views: 303

Q View related articles $\llbracket$

View Crossmark data $₫$ 


\title{
A Counting Proof for When \\ 2 Is a Quadratic Residue
}

\section{Karthik Chandrasekhar, Richard Ehrenborg, and Frits Beukers}

\begin{abstract}
Using the group consisting of the eight Möbius transformations $x,-x, 1 / x,-1 / x$, $(x-1) /(x+1),(x+1) /(1-x),(x+1) /(x-1)$, and $(1-x) /(x+1)$, we present an enumerative proof of the classical result for when the element 2 is a quadratic residue in the finite field $F_{q}$.
\end{abstract}

Recall that a nonzero element $x$ in a field $F$ is a quadratic residue if it is a square, that is, we can write $x=y^{2}$ where $y \in F$.

Assume that $q$ is an odd prime power and let $F_{q}$ be the finite field of $q$ elements. The classical result that -1 is a quadratic residue in $F_{q}$ if and only if $q \equiv 1 \bmod 4$ can be proved by partitioning the nonzero elements of the field into orbits of the form $\{x,-x,-1 / x, 1 / x\}$. Note that one orbit is $\{1,-1\}$. If $\alpha^{2}=-1$ has a solution, then $\{\alpha,-\alpha\}$ is also an orbit. The remaining orbits all have cardinality 4 . Thus by counting the nonzero elements of the field modulo 4 , we obtain that $q \equiv 1 \bmod 4$, implying that $q-1 \equiv 0 \equiv|\{1,-1\}|+|\{\alpha,-\alpha\}| \bmod 4$ and hence that the orbit $\{\alpha,-\alpha\}$ exists, that is, -1 is a quadratic residue. Similarly, $q \equiv 3 \bmod 4$ implies that there is no such orbit and hence -1 is not a quadratic residue. See [1, Theorem 2.2.7].

We present a similar argument for when the element 2 is a quadratic residue. We use a larger set of rational functions and we have four different types of orbits.

Theorem 1. Let $q$ be an odd prime power. Then the element 2 is a quadratic residue in the finite field $F_{q}$ if and only if $q \equiv \pm 1 \bmod 8$.

Proof. Consider the eight rational functions $x,-x, 1 / x,-1 / x,(x-1) /(x+1),(x+$ $1) /(1-x),(x+1) /(x-1)$, and $(1-x) /(x+1)$. Note that they form a group $G$ under composition. These rational functions are Möbius transformations and act naturally on the field $F_{q}$ with the point at infinity adjoined, that is, on $F_{q} \cup\{\infty\}$. The orbits of this action are as follows. First there is the orbit $\{0, \pm 1, \infty\}$. In fact, the group permutes these elements as the vertices of a square, showing that the group is isomorphic to the symmetric group of a square. Assuming that 2 is a quadratic residue in the field $F_{q}$, we have the orbit $B=\{ \pm 1 \pm \sqrt{2}\}$ of size 4 . Next, assuming that -1 is a quadratic residue, we have the orbit $C=\{ \pm i\}$ of size 2 . Finally, the remaining orbits all have size 8 .

We now have four cases. In each case, it is enough to count the $q-3$ elements in $F_{q}-\{0, \pm 1\}$ modulo 8 , hence only keeping track if the orbits $B$ and $C$ occur.

- If -1 and 2 are both quadratic residues, then both $B$ and $C$ occur, yielding $q-3 \equiv$ $4+2 \bmod 8$, that is, $q \equiv 1 \bmod 8$.

- If -1 and 2 are both not quadratic residues, then all orbits have size 8 , yielding $q-3 \equiv 0 \bmod 8$, that is, $q \equiv 3 \bmod 8$.

doi.org/10.1080/00029890.2020.1790925

MSC: Primary 11A07 
- If -1 is a quadratic residue and 2 is not, then only $C$ occurs, yielding $q-3 \equiv$ $2 \bmod 8$, that is, $q \equiv 5 \bmod 8$.

- Finally, if 2 is a quadratic residue and -1 is not, then only $B$ occurs, yielding $q-3 \equiv 4 \bmod 8$, that is, $q \equiv 7 \bmod 8$.

A similar proof can be obtained by using the order 6 group $H=\{x, 1-x, 1 /(1-$ $x), x /(x-1),(x-1) / x, 1 / x\}$. When $q \equiv 3 \bmod 4$, the result follows by counting the number of quadratic residues in orbits of $H$. Similarly, when $q \equiv 1 \bmod 4$, the result follows by counting the number of quadratic nonresidues.

ACKNOWLEDGMENTS. The authors thank David Leep for suggestions that improved the exposition of an earlier version of this note. This work was partially supported by a grant from the Simons Foundation (\#429370 to Richard Ehrenborg).

\section{REFERENCE}

[1] Davidoff, G., Sarnak, P., Valette, A. (2003). Elementary Number Theory, Group Theory, and Ramanujan Graphs. Cambridge, UK: Cambridge Univ. Press.

Department of Mathematics, University of Kentucky, Lexington, KY, USA

ak.c@uky.edu

Department of Mathematics, University of Kentucky, Lexington, KY, USA

richard.ehrenborg@uky.edu

Department of Mathematics, University of Utrecht, 3508 TA Utrecht, The Netherlands f.beukers@uu.nl 